\title{
Parkinson's in the bone
}

\author{
Lei Xiong ${ }^{1,2}$, Jin-Xiu Pan ${ }^{1,2}$, Hao-han Guo ${ }^{1}$, Lin Mei ${ }^{1,2}$ and Wen-Cheng Xiong ${ }^{1,2^{*}}$ (])
}

\begin{abstract}
Patients with Parkinson's disease (PD) exhibit systemic deficits, including arthritis and osteoporosis-like symptoms. However, the questions, how the deficits in periphery organs or tissues occur in PD patients, and what are the relationship (s) of the periphery tissue deficits with the brain pathology (e.g., dopamine neuron loss), are at the beginning stage to be investigated. Notice that both PD and osteoporosis are the products of a complex interaction of genetic and environmental risk factors. Genetic mutations in numerous genes have been identified in patients either with recessive or autosomal dominant PD. Most of these PD risk genes are ubiquitously expressed; and many of them are involved in regulation of bone metabolism. Here, we review the functions of the PD risk genes in regulating bone remodeling and homeostasis. The knowledge gaps in our understanding of the bone-to-brain axis in PD development are also outlined.
\end{abstract}

Keywords: Parkinson's disease, Bone homeostasis, Osteoporosis

\section{Introduction}

Parkinson's disease (PD) is the 2nd most common neurodegenerative disease, clinically characterized as a movement disorder. Since Dr. James Parkinson initially describes the symptoms of "Shaking Palsy", and Dr. Charcot re-named it as "Parkinson's Disease" (PD) [1-3], nearly two centuries have passed. Until now, PD remains to be a terminal degenerative disease without effective cure therapy. Interestingly, PD was considered as a nongenetic disease long time ago, because epidemiological studies showed evidence to link PD with the environmental factors such as neurotoxins or viral infection [4, 5]. For examples, drug users exposed to 1-methyl-4-phenyl-1,2,5,6-tetrahydropyridine (MPTP) show parkinsonian-like features, and a pandemic influenza virus is found to be strongly associated with post-encephalitic parkinsonism [6]. In 1996, it marked the starting point for PD genetic studies, which mapped and subsequently identified mutations in SNCA ( $\alpha$-synuclein) or PARK1 gene to be responsible for PD $[7,8]$. In the following

*Correspondence: Wen-Cheng.Xiong@case.edu

${ }^{1}$ Department of Neurosciences, School of Medicine, Case Western Reserve University, Cleveland, $\mathrm{OH} 44106$, USA

Full list of author information is available at the end of the article years, additional mutations related to PD, such as PINK1, PRKN, LRRK2, and VPS35, have been identified $[9,10]$. To date, more than 20 autosomal dominant and recessive genetic mutations have been found to be involved in the pathogenesis of PD [11, 12].

It is also of interest to note that PD was considered as a disease with systemic deficits, including arthritic joint pain, constipation, muscle weakness and rigidity, and increased incidence of osteoporosis and bone fractures, according to Drs. Parkinson and Charcot' initial descriptions $[2,3]$. However, since the discovery of the dopamine neuron loss in PD patients' brain in 1960s [13], the central dopamine hypothesis has been dominant in the field. Recent molecular, biological, and genetic studies suggest that the PD risk genes are expressed not only in the brain, but also in periphery organs/tissues, including bone cells; and mouse models expressing or suppressing the PD risk gene could exhibit phenotypes not only in the brain, but also in periphery organs/tissues $[14,15]$. These observations have thus led scientists to re-think the view that PD is a systemic disorder.

Bone remodeling is a dynamic process essential for maintenance of skeletal integrity and bone homeostasis, in which old bone is destructed by bone-resorbing osteoclasts (OCs) and subsequently replaced with new original author(s) and the source, provide a link to the Creative Commons licence, and indicate if changes were made. The images or other third party material in this article are included in the article's Creative Commons licence, unless indicated otherwise in a credit line to the material. If material is not included in the article's Creative Commons licence and your intended use is not permitted by statutory regulation or exceeds the permitted use, you will need to obtain permission directly from the copyright holder. To view a copy of this licence, visit http://creativecommons.org/licenses/by/4.0/. The Creative Commons Public Domain Dedication waiver (http://creativeco mmons.org/publicdomain/zero/1.0/) applies to the data made available in this article, unless otherwise stated in a credit line to the data. 
bone by bone-forming osteoblasts (OBs) [16]. The imbalance of bone formation and resorption could result in metabolic bone diseases such as osteoporosis. PD and osteoporosis are two conditions affecting a substantial portion of the elderly population [17]. PD patients usually have bone and joint problems, and have a high risk for fractures, which are believed to be induced by falls due to decreased mobility, postural instability, neurological impairment and reduced bone mass [18]. Both PD and osteoporosis are the product of a complex interaction of genetic and environmental factors. Many genetic mutations identified in PD patients have been found to regulate not only PD's brain pathology, but also bone metabolism (Table 1). Below, we summarize the functions of these PD risk genes in regulating bone remodeling and bone homeostasis.

\section{Autosomal recessive PD risk genes in autophage, mitochondrial function, and bone homeostasis PINK1 and PRKN}

PINK1, stands for PTEN induced putative kinase 1 (PINK1), is also called PARK6 [19]. PRKN, also named as Parkin or PARK2, is an E3 ubiquitin ligase [20]. Genetic studies have identified mutations in both PINK1 and PRKN genes in patients with recessive PD [21, 22]. Cell biological studies have suggested that both proteins form a complex, and PRKN is one of substrates of PINK1 [20]; defects in PINK1 and Parkin cause an impairment in mitophage mediated clearance of damaged mitochondria, and thus an accumulation of dysfunctional mitochondria, leading to the loss of DA neurons with age [23].

Interestingly, PINK1 and Parkin-mediated mitophagy is reported to play a protective role in bone impairment induced by aluminum exposure [24]. Parkin-deficient mice exacerbated bone impairment, mitochondrial damage, and oxidative stress under aluminum exposure [24]. Zhang et al. have found that parkin could meditate osteoblastic differentiation of BMSCs via $\beta$-catenin and autophagy signaling [25]. Upregulation of parkin could promote $\beta$-catenin expression and autophagy, enhancing expression of osteo-specific markers [25]. Moreover, parkin-overexpression accelerates bone healing in a tibial fracture model [25]. Furthermore, it is reported that p53 and parkin co-regulate mitophagy in bone marrow mesenchymal stem cells to promote the repair of early steroid-induced osteonecrosis of the femoral head (ONFH) [26].

\section{PARK7 (DJ-1)}

PARK7 also known as DJ-1, is a 189 amino acid protein that is widely expressed in different organs/tissues [27]. PARK7 is linked to an early onset of autosomal recessive PD via homozygous deletion and loss of function mutation of PARK7 gene [28]. Cell biological studies have suggested PARK7's function in regulating mitochondrial function and preventing ROS (free oxygen species) production $[29,30]$.

Notably, a recent study, by Kim et al., has uncovered a pivotal role of PARK7 in regulating bone homeostasis [31]. PARK7 (DJ-1) appears to play a critical role in negative regulation of osteoclastogenesis, as well as bone-associated pathology [31]. PARK7 deficient mice exhibit a decrease in bone volume, but an increase in OC numbers, which are likely due to the increased intracellular ROS concentration, and RANKL-induced signaling [31]. In addition to its effects on osteoclastogenesis, PARK7 has been found to stimulate the differentiation of human mesenchymal stem cells to osteoblasts; and to induce angiogenesis in endothelial cells through activation of fibroblast growth factor receptor-1 signalling [32]. Extracellular application of PARK7 could enhance bone regeneration by stimulating the formation of blood vessels and new bones in the rodent model of bone fracture repair [32].

\section{Autosomal recessive PD risk genes in protein ubiquitination, degradation, and bone homeostasis UCHL 1 (PARK5)}

Ubiquitin C-terminal hydrolase L1 (UCHL1) is a component of the ubiquitin proteasome system. Despite the controversy, UCHL1 S18Y variant is reported to be a risk factor for PD [33-36]. Shim et al. found that UCHL1 is expressed in osteoblasts, osteoclasts, and hematopoietic precursor cells of bone marrow in the metaphysis and diaphysis of the femora. In addition, gracile axonal dystrophy (gad) mice, which lack UCHL1 expression, shown reduced bone mineral density (BMD) rate in the metaphysis and diaphysis of the femora, suggest a critical role of UCHL1 in regulating bone mineralization during osteogenesis [37].

\section{FBXO7}

As a member of the F-box protein (FBP) family, FBXO7 (F-box only protein 7) is a substrate-recognition component of Skp1-cullin-F-box protein ubiquitin E3 ligase, which function in phosphorylation-dependent ubiquitination [38]. Mutations in FBXO7 gene are identified in patients closely associated with progression of the autosomal recessive form of familial PD [39].

$\mathrm{FBXO7}$ is reported to affect bone morphoenetic protein 4 (BMP4)-mediated signaling through proteasomeindependent ubiquitination of NRAGE and augments formation of downstream signaling components [40]. However, this study is mainly carried out in vitro, and it remains to be investigated whether it affects bone remodeling and homeostasis in vivo. 


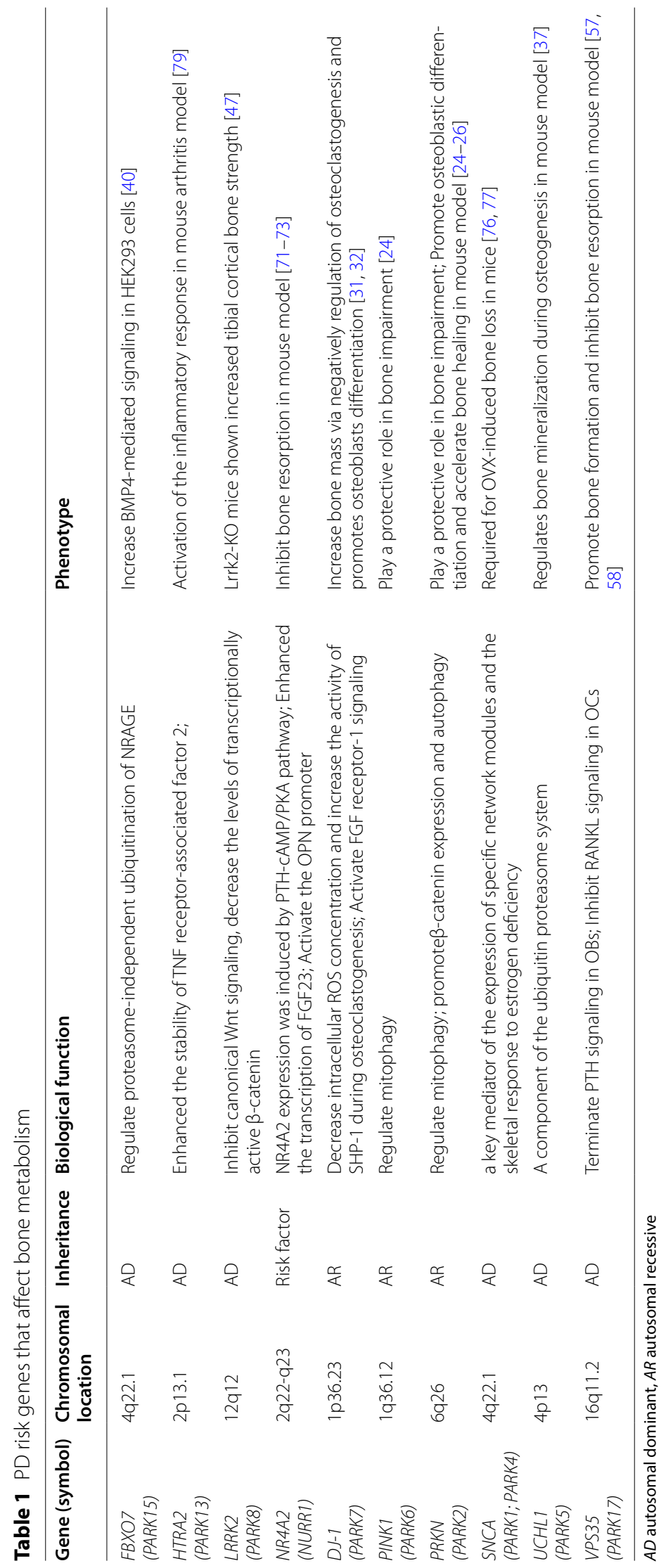




\section{Autosomal dominant PD risk genes in Wnt/ $\beta$-catenin signaling and bone homeostasis $W n t / \beta$-catenin signaling}

The canonical Wnt/ $\beta$-catenin signaling is essential for regulating bone-mass homeostasis $[16,41]$. The secretion of Wnt depends on the wntless receptor [42-44] (Fig. 1). The binding of Wnt ligands to a dual-receptor complex of frizzled and Lrp5/6 results in the accumulation of cytoplasmic $\beta$-catenin and translocation of $\beta$-catenin into the nucleus to regulate gene expression (Fig. 1). This pathway is necessary for commitment of mesenchymal stem cells to the OB lineage, $\mathrm{OB}$ precursor cell proliferation and differentiation, and $\mathrm{OC}$ genesis and activation [16, 41, 45].

\section{LRRK2 (PARK8)}

Leucine rich repeat kinase 2 (LRRK2) mutations account for up to $40 \%$ of $\mathrm{PD}$ cases in some populations, and elicit symptoms and brain pathologies resembling idiopathic PD [46]. By using Lrrk2 knockout mice, Berwick et al. investigated the effect of loss of Lrrk2 on canonical Wnt signaling in vitro and in vivo. They found that loss of Lrrk2 causes a dose-dependent increase in the levels of transcriptionally active $\beta$-catenin in the brain, while over-expressed LRRK2 binds and represses $\beta$-catenin, suggesting Lrrk2 may act as part of the $\beta$-catenin destruction complex. Lrrk2-KO could also increase tibial cortical bone strength so that alters tibial bone architecture, decreasing the predicted risk of fracture
[47]. Interestingly, many Lrrk2 mutants identified in PD patients appear to gain of LRRK2's functions [48, 49], and thus suppressing wnt/ $\beta$-catenin signaling (Fig. 1B).

\section{VPS35 (PARK17)}

The vacuolar protein sorting ortholog 35 (VPS35) is a key component of the retromer, which is responsible for selective retrieval of transmembrane cargo proteins from endosome to trans-Golgi apparatus (Fig. 1A). VPS35 is also called PARK 17, because mutations in Vps35/Park 17 locus, such as D620N and R524W, have been unambiguously identified to cause PD in multiple individuals and families worldwide [50-52]. Thus, dysfunctional VPS35/ retromer has been recently emerged as a new risk factor for late-onset, autosomal dominant familial PD.

Notice that VPS35/retromer is widely expressed in many tissues and cell types, not only in dopamine neurons [53-56], but also in bone cells, such as osteoblasts and osteoclasts [57, 58]. Vps35 hemizygous deficient mice exhibit osteoporotic deficits with increased bone resorption and decreased bone formation $[57,58]$. Interestingly, Xia et al. have found that RANK (receptor activator of NF-kB) in macrophages or osteoclast-lineage cells is a cargo protein of VPS35/retromer [57]; and loss of VPS35's function in macrophages or osteoclast-lineage cells results in increased RANK surface distribution, enhanced RANKL sensitivity, sustained RANKL-RANK

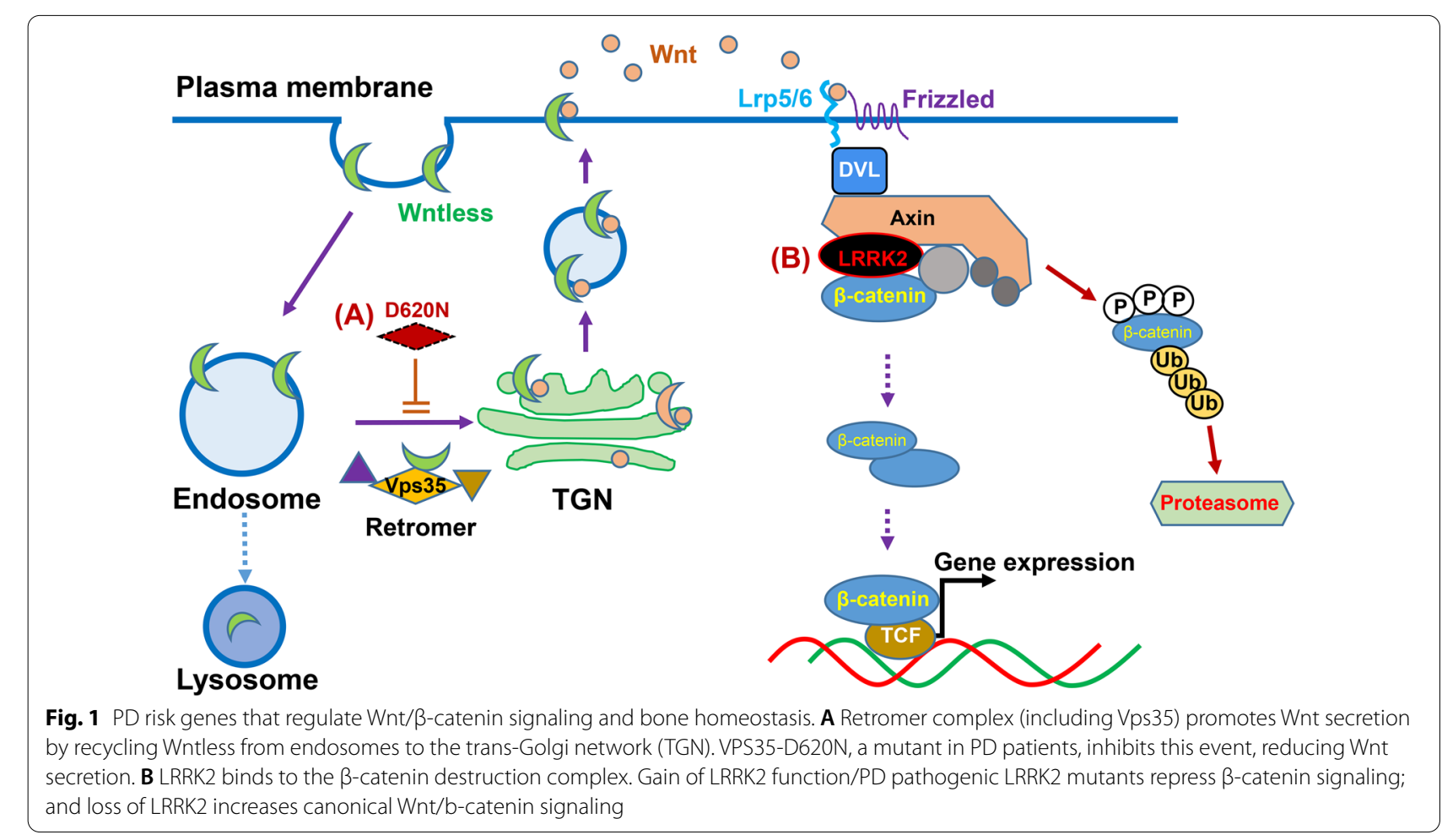


signaling, and thus increasing hyper-resorptive osteoclast formation [57].

In addition to the increased osteoclast mediated bone resorption, mice with Vps35 hemizygous deficiency or the osteoblast selective VPS35 conditionally knocking out display decreased osteoblast mediated bone formation $[57,58]$, another key cellular process underlying the osteoporotic bone loss. In light of numerous reports that demonstrate that Wntless, a sorting receptor, work together with the retromer complex to promote the secretion of Wnt family proteins [59-63] (Fig. 1); and the loss of Wntless impairs Wnt secretion, reducing Wnt/bcatenin signaling and bone formation $[64,65]$, it is likely that VPS35 regulating Wntless trafficking and function in osteoblast-lineage cells may account for their function in promoting osteoblast mediated bone formation. This view is in line with a report by Chiu et al., who have found that the VPS35-D620N mutation causes the malfunction of Vps35 and impairs activity of Wnt/ $\beta$-catenin pathway in substantia nigra pars compacta dopaminergic neurons [66] (Fig. 1A). However, this view requires further investigation in osteoblast lineage cells.

\section{PD risk genes in PTH signaling and bone homeostasis PTH signaling}

The recombinant parathyroid hormone (PTH) is the only therapy for postmenopausal osteoporosis that increase bone mass. Intermittent treatment with $\mathrm{PTH}[1-34]$ promotes recruitments of both osteoblasts and osteoclasts, resulting in a net bone-gain, but its continued treatment leads to more osteoclast activation with a net bone-loss [67]. Binding of PTH and PTH receptor (PTH1R) initiates intracellular cyclic adenosine monophosphate (cAMP) signaling that is dynamically regulated [68] (Fig. 2). Below, we highlight the PD risk genes' function in regulating PTH signaling and bone homeostasis.

\section{VPS35}

Notice that in addition to Wntless and RANK, PTH1R is also identified as a cargo of VPS35/retromer, and the PTH1R signaling is dynamically regulated by the retromer complexes [58] (Fig. 2A). Vps35 knockdown in kidney cells [68] and osteoblasts [58] resulted in a slight elevation and extended cAMP response to PTH. Xiong et al. further investigate VPS35's function in regulating

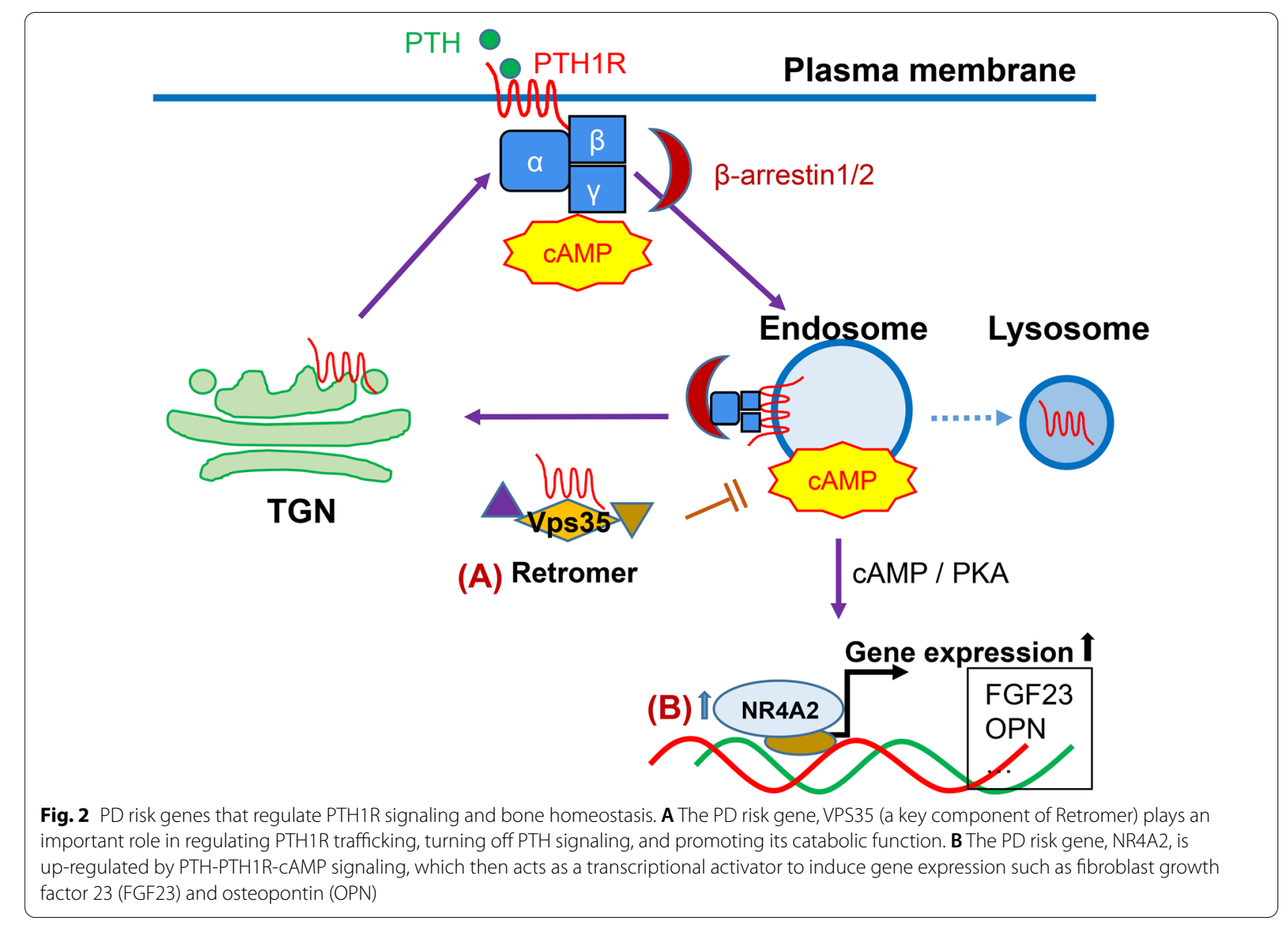


PTH signaling in vivo. Specific Vps35 knockdown in the osteoblast lineage cells, using osteocalcin-targetedCre, results in mildly lowered bone mass in the primary spongiosa, but show a greater increase in bone mass in the primary spongiosa in response to $\mathrm{PTH}$, as compared to controls [58]. They further provide evidence for Vps35 to regulate PTH1R trafficking, to turn off PTH signaling, and to promote its catabolic function in culture and in mice [58]. Additionally, Xiong et al. have identified phosphatase 1 regulatory subunit 14C (PPP1R14C), an inhibitory subunit of PP1 phosphatase, as a VPS35 binding partner, to be involved in VPS35/retromer termination of the endosomal PTH1R signaling [58]. However, whether and how VPS35 mutants (e.g., D620N) alter PTH signaling and bone homeostasis remain to be investigated.

\section{NR4A2 (Nurr1)}

Nuclear receptor subfamily 4 group A member 2 (NR4A2), also known as Nurr1, is a member of the nuclear receptor family of intracellular transcription factors [69]. It is mainly expressed in the central nervous system, but it is also detectable in peripheral tissues such as bone cells. NR4A2 is involved in the pathogenesis of diseases that need dopamine transmission such as PD [70]. Tetradis et al. found that PTH could induce the expression of NR4A2 in primary mouse osteoblasts, which is mediated primarily through the cAMP/PKA pathway [71] (Fig. 2B). The increased NR4A2 then enhances the PTH-induced transcription of FGF23 (fibroblast growth factor 23), a critical growth factor for skeleton development [72]. NR4A2 is also found to mediate PTH-induced expression of osteopontin (OPN) [73]. NR4A2 appears to be transcriptional activator for OPN expression, as it binds and activates the OPN promoter in osteoblastic cells in a synergistic fashion (Fig. 2B), and thus regulate bone homeostasis [73].

\section{Other autosomal dominant PD risk genes in bone homeostasis \\ SNCA}

Alpha-synuclein is a small 140 amino polypeptide encoded by SNCA gene that is a major component of Lewy body (LB) inclusions in PD patients [74, 75]. Alphasynuclein is highly expressed in neural tissue, osteoblasts, erythroblasts, macrophages, and adipose tissue [76]. As an autosomal dominant gene, SNCA was found to regulate bone network homeostasis and ovariectomy-induced bone loss [76]. Calabrese et al. used a mouse model of postmenopausal bone loss (ovariectomy-induced bone loss) and variation in gene expression generated by the divergent genetic backgrounds of inbred mouse strains to construct a bone co-expression network in intact and ovariectomized mice, they identified a module of genes whose expression is associated with OVX-induced bone loss, and alpha-synuclein (Snca), is a key mediator of the expression of specific network modules and the skeletal response to estrogen deficiency. In addition, Snca deficiency protects mice from OVX-induced bone loss, the bone loss due to OVX was significantly less in $\mathrm{Snca}^{-1-}$ mice than littermate controls [76]. However, using the Prrx1Cre to conditionally delete Snca in osteoprogenitor cells, Figueroa et al. show that deletion of Snca in Prrx$\mathrm{Cre}(+)$ cells causes partial loss of function in the central nervous system but does not affect OVX-induced bone loss [77], implicating that Snca may regulate bone remodeling in an indirect manner. Further analysis is necessary to address this issue.

\section{HTRA2 (OMI; PARK13)}

HtrA serine peptidase 2 (HTRA2), also known as OMI, is a mitochondrially-located serine protease which is involved in PD [78]. Rheumatoid arthritis (RA) is an autoimmune inflammatory disease characterized by the destruction of cartilage and bone. HtrA2 was reported to participated in the activation of the inflammatory response in a collagen-induced arthritis model. HtrA2 modulated inflammatory responses in bone marrowderived macrophages (BMDMs) by controlling TNF receptor-associated factor 2 (TRAF2) stability in a collagen-induced arthritis mouse model [79].

\section{Conclusion and future questions}

Osteoporosis in PD patients has not been extensively studied. On one hand, decreased mobility, abnormal posture, as well as falling increase the risk of fractures. On the other hand, many PD risk genes have been found to be directly involved in the regulation of bone remodeling. Further investigating this issue may reveal new insights into the strategy developments for the clinical diagnosis and treatment of PD. A clinical fracture risk evaluation and bone densitometry to detect osteoporosis in newly identified PD patients may be useful for our further understanding their association. The medication used for the long-term treatments of PD may need safety studies for bone preservation, as we do not know whether these medications have any effect on bone health. PD and osteoporosis risk genes may have similar mechanisms in pathogenicity. However, further investigating their functions in bone may be helpful in the design of the new targeted drug development that can simultaneously treat both PD and osteoporosis.

Notice that many PD risk genes (such as ATP13A2, EIF4G1, GBA, VPS13C, and so on)' functions in bone remodeling and homeostasis have not been investigated, although they are widely expressed in various tissues and have important regulatory effects on cell 
metabolism such as autophagy, immune response, mitochondrial biology, lysosomal dysfunction (e.g. $G B A, A T P 13 A 2)$ and endocytic pathway [80, 81]. For example, the $G B A$ gene encodes a lysosomal enzyme $\beta$-glucocerebrosidase with an important role in glycolipid metabolism. Loss-of-function mutations in $\beta$-glucocerebrosidase cause an accumulation of glucocerebroside that results in a wide spectrum of symptoms involving the liver, blood, bone marrow, spleen, lungs, and the nervous system, known as Gaucher disease [82]. Gaucher disease can weaken bone, increasing the risk of painful fractures. It can also interfere with the blood supply to your bones, which can cause portions of the bone to die [83]. Thus, in addition to brain, it is of importance to investigate the functions of these genes in periphery tissues or organs, including bone.

\begin{abstract}
Abbreviations
BMD: Bone mineral density; BMDMs: Bone marrow-derived macrophages; BMP4: Bone morphoenetic protein 4; BMSCs: Bone marrow stem/stromal cells; CAMP: Cyclic adenosine monophosphate; FBXO7: F-box only protein 7; FGF23: Fibroblast growth factor 23; HTRA2: HtrA serine peptidase 2; LB: Lewy body; LRRK2: Leucine rich repeat kinase 2; MPTP: 1-Methyl-4-phenyl-1,2,5,6-tetrahydropyridine; NR4A2: Nuclear receptor subfamily 4 group A member 2; OBs: Osteoblasts; OCs: Osteoclasts; ONFH: Osteonecrosis of the femoral head; PD: Parkinson's disease; PINK1: PTEN induced putative kinase 1; PPP1R14C: Phosphatase 1 regulatory subunit 14C; PTH: Parathyroid hormone; RA: Rheumatoid arthritis; RANK: Receptor activator of NF-KB; ROS: Free oxygen species; TRAF2: TNF receptor-associated factor 2; UCHL1: Ubiquitin C-terminal hydrolase L1; VPS35: Vacuolar protein sorting ortholog 35.
\end{abstract}

\section{Acknowledgements}

We thank all the members of Xiong lab and Mei lab for their helps and suggestions.

\section{Authors' contributions}

LX and WCX write the manuscript. JP, HG, and LM provide some suggestions. All authors read and approved the final manuscript.

\section{Funding}

Not applicable.

\section{Availability of data and materials}

Not applicable.

\section{Declarations}

Ethics approval and consent to participate

Not applicable.

\section{Consent for publication}

Not applicable.

\section{Competing interests}

The authors declare that they have no competing interests.

\section{Author details}

${ }^{1}$ Department of Neurosciences, School of Medicine, Case Western Reserve University, Cleveland, OH 44106, USA. ${ }^{2}$ Louis Stoke VA Medical Center, Cleveland, $\mathrm{OH} 44106$, USA.

Received: 12 October 2021 Accepted: 26 October 2021

Published online: 05 November 2021

\section{References}

1. Horowski R, Horowski L, Vogel S, Poewe W, Kielhorn FW. An essay on Wilhelm von Humboldt and the shaking palsy: first comprehensive description of Parkinson's disease by a patient. Neurology. 1995;45:565-8.

2. J. Parkinson, An essay on the shaking palsy. 1817. J Neuropsychiatry Clin Neurosci 14, 223-236; discussion 222 (2002).

3. Goetz CG. The history of Parkinson's disease: early clinical descriptions and neurological therapies. Cold Spring Harb Perspect Med. 2011;1:a008862.

4. Duvoisin RC, Eldridge R, Williams A, Nutt J, Calne D. Twin study of Parkinson disease. Neurology. 1981;31:77-80.

5. Eldridge R, Ince SE. The low concordance rate for Parkinson's disease in twins: a possible explanation. Neurology. 1984;34:1354-6.

6. Langston JW, Ballard PA Jr. Parkinson's disease in a chemist working with 1-methyl-4-phenyl-1,2,5,6-tetrahydropyridine. N Engl J Med. 1983;309:310.

7. Polymeropoulos MH, et al. Mapping of a gene for Parkinson's disease to chromosome 4q21-q23. Science. 1996;274:1197-9.

8. Polymeropoulos $\mathrm{MH}$, et al. Mutation in the alpha-synuclein gene identified in families with Parkinson's disease. Science. 1997;276:2045-7.

9. Kitada T, et al. Mutations in the parkin gene cause autosomal recessive juvenile parkinsonism. Nature. 1998;392:605-8.

10. Zimprich A, et al. Mutations in LRRK2 cause autosomal-dominant parkinsonism with pleomorphic pathology. Neuron. 2004;44:601-7.

11. Deng H, Wang P, Jankovic J. The genetics of Parkinson disease. Ageing Res Rev. 2018;42:72-85.

12. Lesage S, Brice A. Parkinson's disease: from monogenic forms to genetic susceptibility factors. Hum Mol Genet. 2009;18:R48-59.

13. Ehringer $\mathrm{H}$, Hornykiewicz $\mathrm{O}$. Distribution of noradrenaline and dopamine (3-hydroxytyramine) in the human brain and their behavior in diseases of the extrapyramidal system. Klin Wochenschr. 1960;38:1236-9.

14. Dawson TM, Ko HS, Dawson VL. Genetic animal models of Parkinson's disease. Neuron. 2010;66:646-61.

15. Lee $Y$, Dawson VL, Dawson TM. Animal models of Parkinson's disease: vertebrate genetics. Cold Spring Harb Perspect Med. 2012;2:1.

16. Baron R, Kneissel M. WNT signaling in bone homeostasis and disease: from human mutations to treatments. Nat Med. 2013;19:179-92.

17. Invernizzi M, Carda S, Viscontini GS, Cisari C. Osteoporosis in Parkinson's disease. Parkinsonism Relat Disord. 2009;15:339-46.

18. Gnadinger M, Mellinghoff HU, Kaelin-Lang A. Parkinson's disease and the bones. Swiss Med Wkly. 2011;141:13154.

19. Valente EM, et al. Hereditary early-onset Parkinson's disease caused by mutations in PINK1. Science. 2004;304:1158-60.

20. Kane LA, et al. PINK1 phosphorylates ubiquitin to activate Parkin E3 ubiquitin ligase activity. J Cell Biol. 2014;205:143-53.

21. Healy DG, et al. PINK1 (PARK6) associated Parkinson disease in Ireland. Neurology. 2004;63:1486-8.

22. Camargos ST, et al. Familial Parkinsonism and early onset Parkinson's disease in a Brazilian movement disorders clinic: phenotypic characterization and frequency of SNCA, PRKN, PINK1, and LRRK2 mutations. Mov Disord. 2009;24:662-6.

23. Pickrell AM, Youle RJ. The roles of PINK1, parkin, and mitochondrial fidelity in Parkinson's disease. Neuron. 2015:85:257-73.

24. Cui Y, et al. PINK1/Parkin-Mediated Mitophagy Plays a Protective Role in the Bone Impairment Caused by Aluminum Exposure. J Agric Food Chem. 2021;69:6054-63.

25. Zhang W, et al. Upregulation of Parkin Accelerates Osteoblastic Differentiation of Bone Marrow-Derived Mesenchymal Stem Cells and Bone Regeneration by Enhancing Autophagy and beta-Catenin Signaling. Front Cell Dev Biol. 2020;8:576104.

26. Zhang F, et al. P53 and Parkin co-regulate mitophagy in bone marrow mesenchymal stem cells to promote the repair of early steroid-induced osteonecrosis of the femoral head. Cell Death Dis. 2020;11:42.

27. Bandopadhyay $R$, et al. The expression of DJ-1 (PARK7) in normal human CNS and idiopathic Parkinson's disease. Brain. 2004;127:420-30.

28. Bonifati $V$, et al. DJ-1( PARK7), a novel gene for autosomal recessive, early onset parkinsonism. Neurol Sci. 2003;24:159-60.

29. Canet-Aviles RM, et al. The Parkinson's disease protein DJ-1 is neuroprotective due to cysteine-sulfinic acid-driven mitochondrial localization. Proc Natl Acad Sci USA. 2004;101:9103-8. 
30. Dolgacheva LP, Berezhnov AV, Fedotova El, Zinchenko VP, Abramov AY. Role of DJ-1 in the mechanism of pathogenesis of Parkinson's disease. J Bioenerg Biomembr. 2019;51:175-88.

31. Kim HS, et al. DJ-1 controls bone homeostasis through the regulation of osteoclast differentiation. Nat Commun. 2017;8:1519.

32. Kim JM, et al. DJ-1 promotes angiogenesis and osteogenesis by activating FGF receptor-1 signaling. Nat Commun. 2012;3:1296.

33. Miyake Y, et al. UCHL1 S18Y variant is a risk factor for Parkinson's disease in Japan. BMC Neurol. 2012;12:62.

34. Ragland M, Hutter C, Zabetian C, Edwards K. Association between the ubiquitin carboxyl-terminal esterase L1 gene (UCHL1) S18Y variant and Parkinson's Disease: a HuGE review and meta-analysis. Am J Epidemiol. 2009:170:1344-57.

35. Tan EK, et al. Case-control study of UCHL1 S18Y variant in Parkinson's disease. Mov Disord. 2006;21:1765-8.

36. Healy DG, et al. UCHL-1 is not a Parkinson's disease susceptibility gene. Ann Neurol. 2006;59:627-33.

37. Shim S, Kwon YB, Yoshikawa Y, Kwon J. Ubiquitin C-terminal hydrolase L1 deficiency decreases bone mineralization. J Vet Med Sci. 2008;70:649-51.

38. Petroski MD, Deshaies RJ. Mechanism of lysine 48-linked ubiquitin-chain synthesis by the cullin-RING ubiquitin-ligase complex SCF-Cdc34. Cell. 2005;123:1107-20.

39. Di Fonzo A, et al. FBXO7 mutations cause autosomal recessive, earlyonset parkinsonian-pyramidal syndrome. Neurology. 2009;72:240-5.

40. Kang J, Chung KC. The F-box protein FBXO7 positively regulates bone morphogenetic protein-mediated signaling through Lys-63-specific ubiquitination of neurotrophin receptor-interacting MAGE (NRAGE). Cell Mol Life Sci. 2015;72:181-95.

41. Day TF, Guo X, Garrett-Beal L, Yang Y. Wnt/beta-catenin signaling in mesenchymal progenitors controls osteoblast and chondrocyte differentiation during vertebrate skeletogenesis. Dev Cell. 2005;8:739-50.

42. Banziger $C$, et al. Wntless, a conserved membrane protein dedicated to the secretion of Wnt proteins from signaling cells. Cell. 2006;125:509-22.

43. Bartscherer K, Pelte N, Ingelfinger D, Boutros M. Secretion of Wnt ligands requires Evi, a conserved transmembrane protein. Cell. 2006;125:523-33.

44. Goodman RM, et al. Sprinter: a novel transmembrane protein required for Wg secretion and signaling. Development. 2006;133:4901-11.

45. Glass DA 2nd, et al. Canonical Wnt signaling in differentiated osteoblasts controls osteoclast differentiation. Dev Cell. 2005;8:751-64.

46. Kumari U, Tan EK. LRRK2 in Parkinson's disease: genetic and clinical studies from patients. FEBS J. 2009;276:6455-63.

47. Berwick DC, et al. Pathogenic LRRK2 variants are gain-of-function mutations that enhance LRRK2-mediated repression of beta-catenin signaling. Mol Neurodegener. 2017;12:9.

48. Berwick DC, Harvey K. LRRK2 functions as a Wnt signaling scaffold, bridging cytosolic proteins and membrane-localized LRP6. Hum Mol Genet. 2012;21:4966-79.

49. Sancho RM, Law BM, Harvey K. Mutations in the LRRK2 Roc-COR tandem domain link Parkinson's disease to Wnt signalling pathways. Hum Mol Genet. 2009;18:3955-68

50. A. Deutschlander, O. A. Ross, Z. K. Wszolek, in GeneReviews((R)), M. P. Adam et al., Eds. (Seattle (WA), 1993).

51. Follett J, et al. Parkinson Disease-linked Vps35 R524W Mutation Impairs the Endosomal Association of Retromer and Induces alpha-Synuclein Aggregation. J Biol Chem. 2016;291:18283-98.

52. Zimprich $A$, et al. A mutation in VPS35, encoding a subunit of the retromer complex, causes late-onset Parkinson disease. Am J Hum Genet. 2011;89:168-75

53. Appel JR, et al. Increased Microglial Activity, Impaired Adult Hippocampal Neurogenesis, and Depressive-like Behavior in Microglial VPS35-Depleted Mice. J Neurosci. 2018;38:5949-68.

54. Tang FL, et al. Coupling of terminal differentiation deficit with neurodegenerative pathology in Vps35-deficient pyramidal neurons. Cell Death Differ. 2020;27:2099-116.

55. Wang CL, et al. VPS35 regulates developing mouse hippocampal neuronal morphogenesis by promoting retrograde trafficking of BACE1. Biol Open. 2012;1:1248-57.
56. Wu KY, et al. Ependymal Vps35 promotes ependymal cell differentiation and survival, suppresses microglial activation, and prevents neonatal hydrocephalus. J Neurosci. 2020;40:3862-79.

57. Xia WF, et al. Vps35 loss promotes hyperresorptive osteoclastogenesis and osteoporosis via sustained RANKL signaling. J Cell Biol. 2013;200:821-37.

58. Xiong $L$, et al. Retromer in osteoblasts interacts with protein phosphatase 1 regulator subunit 14C, terminates parathyroid hormone's signaling, and promotes its catabolic response. EBioMedicine. 2016;9:45-60.

59. Belenkaya TY, et al. The retromer complex influences Wnt secretion by recycling wntless from endosomes to the trans-Golgi network. Dev Cell. 2008;14:120-31.

60. Franch-Marro $X$, et al. Wingless secretion requires endosome-to-Golgi retrieval of Wntless/Evi/Sprinter by the retromer complex. Nat Cell Biol. 2008;10:170-7.

61. Pan $\mathrm{CL}$, et al. C. elegans AP-2 and retromer control Wnt signaling by regulating mig-14/Wntless. Dev Cell. 2008;14:132-9.

62. Port $F$, et al. Wingless secretion promotes and requires retromer-dependent cycling of Wntless. Nat Cell Biol. 2008;10:178-85.

63. Yang PT, et al. Wnt signaling requires retromer-dependent recycling of MIG-14/Wntless in Wnt-producing cells. Dev Cell. 2008;14:140-7.

64. Zhong Z, et al. Wntless functions in mature osteoblasts to regulate bone mass. Proc Natl Acad Sci U S A. 2012;109:E2197-2204.

65. Zhong ZA, et al. Wntless spatially regulates bone development through beta-catenin-dependent and independent mechanisms. Dev Dyn. 2015;244:1347-55.

66. Chiu CC, et al. (D620N) VPS35 causes the impairment of Wnt/beta-catenin signaling cascade and mitochondrial dysfunction in a PARK17 knockin mouse model. Cell Death Dis. 2020;11:1018.

67. Frolik CA, et al. Anabolic and catabolic bone effects of human parathyroid hormone (1-34) are predicted by duration of hormone exposure. Bone. 2003;33:372-9.

68. Feinstein TN, et al. Retromer terminates the generation of CAMP by internalized PTH receptors. Nat Chem Biol. 2011;7:278-84.

69. Decressac M, Volakakis N, Bjorklund A, Perlmann T. NURR1 in Parkinson disease-from pathogenesis to therapeutic potential. Nat Rev Neurol. 2013;9:629-36.

70. Le WD, et al. Mutations in NR4A2 associated with familial Parkinson disease. Nat Genet. 2003;33:85-9.

71. Tetradis S, Bezouglaia O, Tsingotjidou A. Parathyroid hormone induces expression of the nuclear orphan receptor Nurr1 in bone cells. Endocrinology. 2001;142:663-70.

72. Meir T, et al. Parathyroid hormone activates the orphan nuclear receptor Nurr1 to induce FGF23 transcription. Kidney Int. 2014;86:1106-15.

73. Lammi J, Huppunen J, Aarnisalo P. Regulation of the osteopontin gene by the orphan nuclear receptor NURR1 in osteoblasts. Mol Endocrinol. 2004;18:1546-57.

74. Maroteaux L, Campanelli JT, Scheller RH. Synuclein: a neuron-specific protein localized to the nucleus and presynaptic nerve terminal. J Neurosci. 1988:8:2804-15.

75. Spillantini MG, et al. Alpha-synuclein in Lewy bodies. Nature. 1997:388:839-40

76. Calabrese G, Mesner LD, Foley PL, Rosen CJ, Farber CR. Network Analysis Implicates Alpha-Synuclein (Snca) in the Regulation of OvariectomyInduced Bone Loss. Sci Rep. 2016;6:29475.

77. Figueroa CA, et al. Deletion of alpha-Synuclein in Prrx 1-positive cells causes partial loss of function in the central nervous system (CNS) but does not affect ovariectomy induced bone loss. Bone. 2020;137:115428.

78. Strauss KM, et al. Loss of function mutations in the gene encoding Omi/ HtrA2 in Parkinson's disease. Hum Mol Genet. 2005;14:2099-111.

79. $\mathrm{Xu} Z$, et al. HtrA2 is required for inflammatory responses in BMDMs via controlling TRAF2 stability in collagen-induced arthritis. Mol Immunol. 2021;129:78-85.

80. Klein C, Westenberger A. Genetics of Parkinson's disease. Cold Spring Harb Perspect Med. 2012;2:a008888.

81. Billingsley KJ, Bandres-Ciga S, Saez-Atienzar S, Singleton AB. Genetic risk factors in Parkinson's disease. Cell Tissue Res. 2018;373:9-20. 
82. Hruska KS, LaMarca ME, Scott CR, Sidransky E. Gaucher disease: mutation and polymorphism spectrum in the glucocerebrosidase gene (GBA). Hum Mutat. 2008;29:567-83.

83. Mikosch P, Hughes D. An overview on bone manifestations in Gaucher disease. Wien Med Wochenschr. 2010;160:609-24.

\section{Publisher's Note}

Springer Nature remains neutral with regard to jurisdictional claims in published maps and institutional affiliations.
Ready to submit your research? Choose BMC and benefit from:

- fast, convenient online submission

- thorough peer review by experienced researchers in your field

- rapid publication on acceptance

- support for research data, including large and complex data types

- gold Open Access which fosters wider collaboration and increased citations

- maximum visibility for your research: over $100 \mathrm{M}$ website views per year

At BMC, research is always in progress.

Learn more biomedcentral.com/submissions 